\title{
A PROBABILISTIC PRIMALITY TEST BASED ON THE PROPERTIES OF CERTAIN GENERALIZED LUCAS NUMBERS
}

\author{
Adina Di Porto and Piero Filipponi \\ Fondazione Ugo Bordoni \\ I-00142 Roma, Italy
}

\begin{abstract}
After defining a class of generalized Fibonacci numbers and Lucas numbers, we characterize the Fibonacci pseudoprimes of the $m^{\text {th }} k i n d$.

In virtue of the apparent paucity of the composite numbers which are Fibonacci pseudoprimes of the $m^{\text {th }}$ kind for distinct values of the integral parameter $m$, a method, which we believe to be new, for finding large probable primes is proposed. An efficient computational algorithm is outlined.
\end{abstract}

\section{Introduction and generalities}

In this paper, after defining the generalized Fibonacci numbers $U_{n}$ and the generalized Lucas numbers $V_{n}$ (Sec.1), the Fibonacci Pseudoprimes of the $m^{\text {th }}$ kind are characterized (Sec.2).

In virtue of the scarceness of the pseudoprimes which are simultaneously of the $m^{\text {th }}$ kind for distinct values of $m$, a method for finding probable primes is proposed in Sec.3 (for a definition of probable primes see [1]).

In Sec. 4 some theoretical aspects concerning the above said pseudoprimes are considered.

Let $m$ be an arbitrary natural number. The generalized Fibonacci numbers $U_{n}(m)$ (or simply $U_{n}$, if there is no fear of confusion) and the generalized Lucas numbers $V_{n}(m)$ (or simply $V_{n}$ ) are defined (e.g., see [2]) by the second order recurrence relations

Work carried out in the framework of the Agreement between the Italian PT Adminisuation and the Fondazione "Ugo Bordoni". 


$$
U_{n+2}=m U_{n+1}+U_{n} ; U_{0}=0, U_{1}=1
$$

and

$$
V_{n+2}=, m V_{n+1}+V_{n} ; V_{0}=2, V_{1}=m,
$$

respectively. These numbers can also be expressed [2] by means of the closed forms (Binet forms)

$$
\begin{aligned}
& U_{n}=\left(\alpha^{n}-\beta^{n}\right) / \Delta, \\
& V_{n}=U_{n-1}+U_{n+1}=\alpha^{n}+\beta^{n},
\end{aligned}
$$

where

$$
\left\{\begin{array}{l}
\Delta=\left(m^{2}+4\right)^{1 / 2} \\
\alpha=(m+\Delta) / 2 \\
\beta=(m-\Delta) / 2
\end{array}\right.
$$

The notations $\alpha_{m}, \beta_{m}$ and $\Delta_{m}$ will be employed whenever the meaning of $\alpha, \beta$ and $\Delta$ can be misunderstood (e.g., see Lemma 2). By (1.5) it can be seen that $\alpha \beta=-1$ and $\alpha+\beta=m$. Moreover, it can be noted that, letting $m=1$ in (1.1) and (1.2), the usual Fibonacci numbers $F_{n}$ and Lucas numbers $L_{n}$ turn out, respectively.

A further interesting expression for $V_{n}$ is [3]

$$
V_{n}=\sum_{i=0}^{[n / 2]} C_{n, i} m^{n-2 i}
$$

where

$$
\left\{\begin{array}{l}
C_{0,0}=2 \\
C_{n, i}=\frac{n}{n-i}\left(\begin{array}{c}
n-i \\
i
\end{array}\right) .
\end{array}\right.
$$

Rewriting (1.6) as

$$
V_{n}=m^{n}+n \sum_{i=1}^{[n / 2]} \frac{C_{n, i}}{n} m^{n-2 i}, \quad(n \geq 1)
$$

noting that, if $n$ is a prime then $C_{n, i} / n$ is an integer and using Fermat's little theorem, the following fundamental property of the numbers $V_{n}$ is established

$$
V_{n}(m) \equiv m(\bmod n) \quad \forall m \quad \text { (if } n \text { is a prime) }
$$




\section{The Fibonacci pseudoprimes of the $m^{\text {th }}$ kind : definition and numerical aspects}

Observing (1.9), the following question arises spontaneously: "Do odd composites exist which satisfy this congruence?" The answer is affirmative.

We define as Fibonacci Pseudoprimes of the $m^{\text {th }}$ kind ( $m$-F.Psps.) all odd composite integers $n$ for which $V_{n}(m) \equiv m(\bmod n)$ and denote them by $s_{k}(m)(k$ $=1,2, \ldots)$. The corresponding sets will be denoted by $S_{m}$, while the sets of all $m$-F.Psps. not exceeding a given $n$ will be denoted by $S_{m, n}$. For example, we found that $s_{1}(1)=705=3 \cdot 5 \cdot 47, s_{1}(2)=169=13^{2}$ and $s_{1}(3)=33=3 \cdot 11$.

The numbers $s_{k}(1)$ have been analyzed in previous papers [4], [5]. In particular, we found that all composite integers belonging to $\mathbb{S}_{1, n}$ (for $n=10^{8}$ ) are square-free and most of them are congruent to 1 both modulo 4 (82.3\%) and modulo 10 (63.2\%). Moreover, we noted that this behavior seems to become more marked as $n$ increases, but we were not able to find any justification of these facts.

Now, another question arises:"Do odd composite integers exist which are $m$-F.Psps. for distinct values of $m$ ?" Once again, the answer is affirmative. For example, the number $34,561=17 \cdot 19 \cdot 107$ is the smallest number belonging to both $S_{1}$ and $S_{2}$.

A computer experiment was carried out essentially to determine the cardinality of the intersections

$$
G_{n, M}=\bigcap_{m=1}^{M} S_{m, n}\left(\begin{array}{l}
n=10^{8} \\
M=1,2, \ldots, \mu\left(\mu: G_{n, \mu}=\varnothing, G_{n, \mu-1} \neq \varnothing\right)
\end{array}\right) .
$$

Namely, we found that, for $n=10^{8}$,

$$
\begin{aligned}
& \left|G_{n, 1}\right|=\left|S_{1, n}\right|=852,\left|G_{n, 2}\right|=48,\left|G_{n, 3}\right|=\left|G_{n, 4}\right|=5, \\
& \left|G_{n, 5}\right|=\left|G_{n, 6}\right|=\left|G_{n, 7}\right|=1,\left|G_{n, 8}\right|=0 .
\end{aligned}
$$

The fact that $\mathbb{G}_{n, 3}$ and $\mathbb{G}_{n, 4}$ have the same cardinality will be justified by Theor.6 (Sec.4). The numbers (below $10^{8}$ ) belonging to these two sets are

$$
\begin{aligned}
s_{89}(1) & =1,034,881=41 \cdot 43 \cdot 587 \\
s_{137}(1) & =2,184,533=13 \cdot 197 \cdot 853 \\
s_{364}(1) & =15,485,185=5 \cdot 79 \cdot 197 \cdot 199 \\
s_{561}(1) & =39,002,041=13 \cdot 19 \cdot 269 \cdot 587 \\
s_{802}(1) & =87,318,001=17 \cdot 71 \cdot 73 \cdot 991
\end{aligned}
$$

of which the latter belongs also to $G_{n, 7}$, besides being a Carmichael number [1]

Let $\sigma_{m}(n)=\left|S_{m, n}\right|$ be the m-F.Psp.-counting function. The behavior of $\sigma_{1}(n)$ vs. $n$ is shown in fig. 1 , while the behavior of $\left|\mathbb{G}_{n, 2}\right|$ is shown in table 1. 


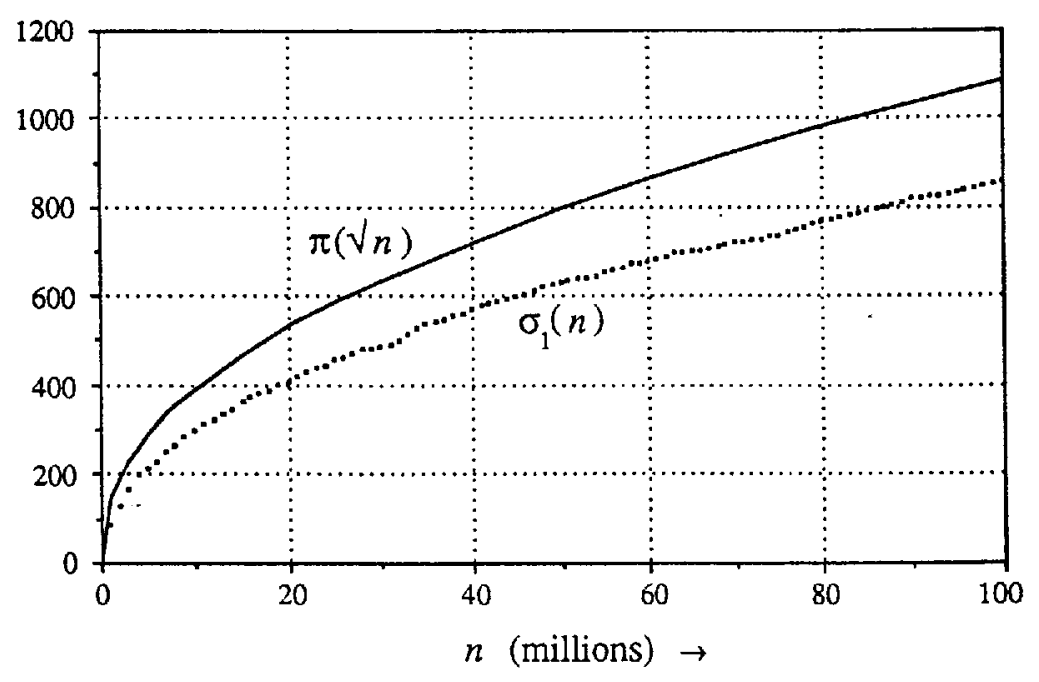

Fig.1- Behavior of $\sigma_{1}(n)$ vs $n$.

Table 1

\begin{tabular}{|c|c|c|c|}
\hline$n$ & $\left|\mathbb{G}_{n, 2}\right|$ & $n$ & $\left|G_{n, 2}\right|$ \\
\hline $10^{7}$ & 18 & $6 \cdot 10^{7}$ & 39 \\
\hline $2 \cdot 10^{7}$ & 27 & $7 \cdot 10^{7}$ & 41 \\
\hline $3 \cdot 10^{7}$ & 30 & $8 \cdot 10^{7}$ & 44 \\
\hline $4 \cdot 10^{7}$ & 36 & $9 \cdot 10^{7}$ & 45 \\
\hline $5 \cdot 10^{7}$ & 38 & $10^{8}$ & 48 \\
\hline
\end{tabular}

Numerically, $\sigma_{1}(n)$ seems asymptotically related to the prime-counting function $\pi(n)$.The inspection of fig. 1 suggests the following

CONJECTURE 1 : "There exists a positive constant $c$ not exceeding 1 such that $\sigma_{1}(n)$ is asymptotic to $c \pi(\sqrt{ } n) . "$

\section{A possible probabilistic primality test}

The numerical evidence that turns out from the experimental results suggests a method for obtaining probable primes.

Let $\langle a\rangle_{b}$ denote the remainder of $a$ divided by $b$. For given integers $n$ (odd) and $M(n>M)$, let us calculate 


$$
r_{m}=\left\langle V_{n}(m)\right\rangle_{n} \quad \text { for } m=1,2, \ldots, M
$$

If $r_{m} \neq m$ for some value of $m$, then $n$ is composite. If $n$ passes $M$ consecutive tests, that is if $r_{m}=m$ for all values of $m(1 \leq m \leq M)$, then $n$ is a probable prime (with probability $P_{M}$ ). A thorough investigation of the properties of the $m$-F.Psps. could suggest a suitable value for $M$ depending on the order of magnitude of $n$. This will be the aim of a future work.

It must be noted that, if Conj. 1 were proved, a sufficiently large $n$ which passes the first test $(m=1)$ would be prime with probability

$$
P_{1} \approx 1-2 c / \sqrt{ } n
$$

Due to the apparent extreme scarceness of the composites $n \in \mathbb{S}_{m}(m=1,2, \ldots, M)$, the probability $P_{M}$ seems to rapidly increase as $M$ increases. The choice of the most suitable set of tests to which submit $n$ is still an open problem.

By suitably modifying the algorithm for obtaining $r_{1}=\left\langle L_{n}\right\rangle_{n}$ [4], an efficient calculation of $V_{n}$ reduced modulo $n$ can be performed. The so-obtained algorithm finds $r_{m}$ after $\left[\log _{2} n\right]$ recursive calculations. For example, ascertaining that the 81-digit composite

$110,221,474,294,665,636,794,016,854,991,608,758,669,691,745,119$.

$008,792,721,304,656,075,481,680,733,031,679$

belongs to $\mathbb{S}_{1}$ required a calculation time of about 25 seconds on a VAX $11 / 750$ computer.

\section{Some properties of the $m$-F.Psps.}

In this section several properties of the $m$-F.Psps. are demonstrated. We hope that they can lead to the discovery of further properties of these numbers. In particular, a formula which gives the minimum value of $M$ (or an upper bound for this value) for which $\left|G_{n, M}\right|=0$, once $n$ is given, would be greatly appreciated.

First, let us state some theorems concerning the case $m=1$.

THEOREM $1:$ If $n$ is an odd integer not divisible by 3 and $L_{n} \equiv 1(\bmod n)$, then

$$
L_{L_{n}} \equiv 1\left(\bmod L_{n}\right) \text {. }
$$

Proof: Since it is known [6] that $L_{n}$ is odd, we can write

$$
L_{n}=4 h \pm 1 \equiv 1(\bmod n) \quad(h \in \mathbb{N}=\{0,1,2, \ldots\}) \text {. }
$$

Case 1: $L_{n}=4 h+1 \equiv 1(\bmod n)$ 
We have $2 h \equiv 0(\bmod n)$, whence [7]

$$
F_{2 h} \equiv 0\left(\bmod L_{n}\right)
$$

From the identities available in $[8, p .95]$, we can write

$$
L_{L_{n}}-1=L_{4 h+1}-1=5 F_{2 h} F_{2 h+1},
$$

whence, by (4.1),

$$
L_{L_{n}}-1 \equiv 5 \cdot 0 \cdot F_{2 h+1} \equiv 0\left(\bmod L_{n}\right) .
$$

Case $2: L_{n}=4 h-1 \equiv 1(\bmod n)$

We have $2 h-1 \equiv 0(\bmod n)$, whence [7]

$$
L_{2 h-1} \equiv 0\left(\bmod L_{n}\right)
$$

Again from $[8, \mathrm{p} .95]$, we can write

$$
L_{L_{n}}-1=L_{4 h-1}-1=L_{2 h} L_{2 h-1}
$$

whence, by (4.3),

$$
L_{L_{n}}-1 \equiv L_{2 h} \cdot 0 \equiv 0\left(\bmod L_{n}\right) . \quad \text { Q.E.D. }
$$

From Theor.1 we can derive the following corollaries.

COROLLARY $1:$ If $p \geq 5$ is a prime and $L_{p}$ is composite, then $L_{p} \in \mathbb{S}_{1}$.

COROLLARY $2:$ If $n$ is not divisible by 3 and belongs to $\mathbb{S}_{1}$, then $L_{n} \in \mathbb{S}_{1}$.

Proof:

(i) From Theor.1 we have $L_{L_{n}} \equiv 1\left(\bmod L_{n}\right)$.

(ii) By hypothesis $n=s t$, with $s$ and $t$ odd integers not divisible by 3 . Hence $L_{n}$ is odd and composite [7]. This completes the proof. It can be noted that also $L_{n}$ is not divisible by 3 , as $n$ is odd [6]. Q.E.D. 
If $n$ is not divisible by 3 and belongs to $S_{1}$, then the number $L_{n}$ fulfils the same conditions. Therefore, we can claim that

$L_{L_{n}} \in \mathbb{S}_{1}$,

and such a statement can be iterated ad infinitum, so that<smiles>[Mg][Te]</smiles>

Consequently, since there exists at least a number $s_{k}(1)$ not divisible by 3 (the smallest among them is $\left.s_{2}(1)=2,465\right)$ the following proposition can be stated

PROPOSITION 1 (Conj. 3 in [4]) : There exist infinitely many 1-F.Psps.

THEOREM $2:$ For $k \in N$,

$$
L_{L_{2^{k}}} \equiv 1\left(\bmod L_{2^{k}}\right) \text {. }
$$

Proof: The statement holds clearly for $k=0,1$. In fact, we have $L_{1} \equiv 1(\bmod 1)$ and $L_{3}$ $\equiv 1(\bmod 3)$. Hence, let us consider $k \geq 2$. It is known [9] that

$$
L_{2^{k}}+1 \equiv 0\left(\bmod 2^{k}\right)
$$

so, $L_{2} k$ can be rewritten as

$$
L_{2^{k}}=h 2^{k}-1 \quad(h \in N)
$$

From (4.6) and $[8$, p.95] we can write

$$
L_{L_{2^{k}}}-1=L_{h 2^{k}-1}-1=L_{4 h 2^{k-2}-1}-1=L_{h 2^{k-1}} L_{h 2^{k-1}-1}
$$

In order to satisfy the congruence

$$
L_{L_{2^{k}}}-1 \equiv 0\left(\bmod L_{2^{k}}\right)
$$

it suffices that the left factor on the right-hand side of (4.7) is divisible by $L_{2^{k}}$, that is, it 
suffices [7] that $h 2^{k-1}$ is an odd multiple of $2^{k}$. Equivalently, we can say that the fulfilment of the equality $h=2(2 t+1)(t \in N)$, that is of the equality (see (4.6))

$$
L_{2^{k}+1}=(2 t+1) 2^{k+1} \quad(t \in \mathbb{N}) \text {, }
$$

is a sufficient condition for the congruence (4.8) to be satisfied.

To establish the general validity of (4.9) we shall use induction on $k$ and the identity $I_{15}[10]$ which allows us to write

$$
L_{2^{k+1}}=L_{2^{k}}^{2}-2
$$

The equality (4.9) holds for $k=2$. In fact, we have $L_{4}+1=8=(2 \cdot 0+1) 2^{3}$. Let us suppose that (4.9) holds up to a certain $k>2$. For the inductive step $k \rightarrow k+1$, from (4.10) and (4.9) we can write

$$
L_{2^{k+1}+1}=L_{2^{k}}^{2}-1=\left[(2 t+1) 2^{k+1}-1\right]^{2}-1=\left(2 t_{1}+1\right) 2^{k+2} \quad\left(t_{1} \in \mathbb{N}\right) .
$$

QE.D.

COROLLARY 3 : If $L_{2^{k}}$ is composite, then $L_{2^{k}} \in \mathbb{S}_{1}$.

To prove the next theorem we need the following

LEMMA 1: If $L_{n} \equiv 0(\bmod n)$, then $L_{n} \equiv 0(\bmod 3 n)$.

Proof: The congruence $L_{n} \equiv 0(\bmod n)$ inplies [8, Theor. F, p.72] that

$$
n=6(2 k+1)=2 \cdot 3^{r+1}(6 h \pm 1) \quad(k, r, h \in N) .
$$

Therefore, it suffices to prove that

$$
L_{n}=L_{2 \cdot 3^{r+1}}(6 h t 1) \equiv 0\left(\bmod 3^{r+2}\right) \text {. }
$$

Let us invoke induction on $r$. The congruence (4.12) holds for $r=0$. In fact, considering the sequence $\left\{L_{n}\right\}$ reduced modulo 9 [6], it is readily seen that $L_{6(6 h \pm 1)}$ $\equiv 0(\bmod 9)$. Let us suppose that $(4.12)$ holds up to a certain $r>0$. For the inductive step $r \rightarrow r+1$, using the identity $L_{s+t}=L_{s} L_{t}-L_{s \rightarrow}$ ( $t$ even) [10], we write

$$
L_{2 \cdot 3^{r+2}(6 h \pm 1)}=L_{(2+1) \cdot 2 \cdot 3^{r+1}(6 h \pm 1)}=L_{2 \cdot 3^{r+1}(6 h \pm 1)}\left(L_{4 \cdot 3^{r+1}(6 h \pm 1)}-1\right) .
$$


It is known [6] that $L_{4 \cdot 3^{r+1}}(6 h \pm 1) \equiv 1(\bmod 3)$. Then, by (4.13) and hypothesis we obtain the congruence $L_{2 \cdot 3^{r+2}(6 h \pm 1)} \equiv 0\left(\bmod 3^{r+3}\right)$. Q.E.D.

THEOREM $3:$ If $L_{n} \equiv 0(\bmod n)$, then

$$
L_{L_{n}-1} \equiv 1\left(\bmod L_{n}-1\right) \text {. }
$$

Proof: Since we have necessarily (see (4.11)) $n=6(2 h+1)$ and, therefore [6] $L_{n}=4 k+2(k \in N)$, from Lemma 1 we have $L_{n}=4 k+2 \equiv 0(\bmod 18(2 h+1))$ $(h \in \mathbb{N})$, that is

$$
2 k+1 \equiv 0(\bmod 9(2 h+1)) .
$$

From $[8, \mathrm{p} .95]$ we can write

$$
L_{n}-1=L_{4(3 h+1)+2}-1=F_{3[2(3 h+1)+1]} / F_{2(3 h+1)+1}
$$

whence

$$
F_{3[2(3 h+1)+1]}=F_{9(2 h+1)} \equiv 0\left(\bmod L_{n}-1\right) .
$$

Again, from $[8$, p.95], we have

$$
L_{L_{n}-1}-1=L_{4 k+1}-1=5 F_{2 k} F_{2 k+1} \text {. }
$$

Since, by (4.16) and (4.14), we see that $L_{n}-1 \mid F_{9(2 h+1)}$ and [7] $F_{9(2 h+1)} \mid F_{2 k+1}$, from (4.17) we obtain

$$
L_{L_{n}-1}-1 \equiv 5 F_{2 k} \cdot 0 \equiv 0\left(\bmod L_{n}-1\right) . \quad \text { Q.E.D. }
$$

COROLLARY $4:$ If $L_{n} \equiv 0(\bmod n)$ and $L_{n}-1$ (necessarily odd) is composite, then

$$
L_{n}-1 \in S_{1} \text {. }
$$

COROLLARY 5 (see [11]): If $L_{2} \cdot 3^{k}-1(k \geq 1)$ is composite, then $L_{2 \cdot 3^{k}}-1 \in S_{1}$.

THEOREM 4: If $n=p_{1} p_{2} \cdots p_{k}$, with $p_{i}=5 h_{i} \pm 1(1 \leq i \leq k)$ is a Carmichael number, then $n \in S_{1}$. 
Proof : Let $P_{i}$ be a repetition period (not necessarily the shortest period) of the Lucas sequence reduced modulo the prime $p_{i}$ and let $\Lambda=\operatorname{l.c.m.}\left(P_{1}, P_{2}, \ldots, P_{k}\right)$.

A sufficient condition for $n$ to belong to $\mathbb{S}_{1}$ is that

$$
h \Lambda+1=n \quad(h \in N) .
$$

In fact, the fulfilment of this condition implies that $L_{h A+1} \equiv L_{1} \equiv 1\left(\bmod p_{1} p_{2} \ldots p_{k}\right)$. On the other hand, it is known [6] that if $p_{i}=5 h_{i} \pm 1$, then $P_{i}=p_{i}-1$. Therefore, it is immediately seen that $\Lambda$ equals the Carmichael $\lambda$ function [1]. Since, by hypothesis, $\Lambda \mid n-1$, from (4.18) the theorem is proved. Q.E.D.

The smallest Carmichael number of the above type which is also a 1-F.Psp. is $s_{44}(1)=252,601=41 \cdot 61 \cdot 101$, while the absolutely smallest Carmichael number which is also a 1-F.Psp. is $s_{2}(1)=2,465=5 \cdot 17 \cdot 29$.

Now, let us state some theorems conceming the case $m \geq 1$.

THEOREM $5:$ If $p \geq 5$ is a prime such that $\Delta^{2}$ is not divisible by $p$, then

$$
V_{U_{p}} \equiv m\left(\bmod U_{p}\right)
$$

Proof: On the basis of the periodicity of the sequence $\left\{U_{n}\right\}$ reduced modulo 4 [6], it can be readily proved that, if $p \geq 5$, then $U_{p}$ has the form $4 h+1(h \in N)$. Since we have $[12] U_{p} \equiv \pm 1(\bmod p)\left(\right.$ except for the case $\Delta^{2} \equiv 0(\bmod p)$ which implies $U_{p} \equiv$ $0(\bmod p))$, we can write $U_{p}=4 h+1 \equiv \pm 1(\bmod p)$.

Case 1: $U_{p}=4 h+1 \equiv 1(\bmod p)$

We have $2 h \equiv 0(\bmod p)$ and, since [12] $U_{n} \mid U_{k n}$,

$$
U_{2 h} \equiv 0\left(\bmod U_{p}\right)
$$

By using the identity

$$
V_{4 h+1}-m=\Delta^{2} U_{2 h} U_{2 h+1}
$$

easily obtainable with the aid of (1.3) and (1.4), we have

$$
V_{U_{p}}-m=V_{4 h+1}-m=\Delta^{2} U_{2 h} U_{2 h+1}
$$

whence, by (4.19) 


$$
V_{U_{p}}-m \equiv \Delta^{2} \cdot 0 \cdot U_{2 h+1} \equiv 0\left(\bmod U_{p}\right)
$$

Case 2: $U_{p}=4 h+1 \equiv-1(\bmod p)$

The proof is analogous to that of Case 1 and is omitted for brevity.

Q.E.D.

It must be noted that, for $m=1$ and $p=5$, the statement of Theor. 5 is true even though $\Delta^{2}=5 \equiv 0(\bmod 5)$. In fact, we have

$$
L_{F_{5}}=L_{5}=11 \equiv 1\left(\bmod F_{5}\right) \text {. }
$$

COROLLARY 6: If $p \geq 5$ is a prime, $\Delta^{2}$ is not divisible by $p$ and $U_{p}$ (necessarily odd) is composite, then $U_{p} \in \mathbb{S}_{m}$.

COROLLARY $7:$ If $p$ is a prime and $F_{p}$ is composite, then $F_{p} \in \mathbb{S}_{1}$.

In order to prove the last theorem, we need to prove the following two lemmata.

LEMMA 2: $V_{n}\left(V_{2 k+1}(m)\right)=V_{n(2 k+1)}(m)$.

Proof: By (1.5) we have

$$
\alpha_{V_{2 k+1}(m)}=\left\{V_{2 k+1}(m)+\left(V_{2 k+1}^{2}(m)+4\right)^{1 / 2}\right\} / 2 .
$$

Using (1.4), (4.22) becomes

$$
\begin{aligned}
\alpha_{V_{2 k+1}(m)}= & \left\{\alpha_{m}^{2 k+1}+\beta_{m}^{2 k+1}+\left(\alpha_{m}^{4 k+2}+\beta_{m}^{4 k+2}+2\right)^{1 / 2}\right\} / 2 \\
& =\left\{\alpha_{m}^{2 k+1}+\beta_{m}^{2 k+1}+\left(\alpha_{m}^{2 k+1}-\beta_{m}^{2 k+1}\right)\right\} / 2=\alpha_{m}^{2 k+1} .
\end{aligned}
$$

Analogously, it is seen that

$$
\beta_{V_{2 k+1}(m)}=\beta_{m}^{2 k+1} .
$$

The statement of the lemma follows directly from (4.23), (4.24) and (1.4). Q.E.D. 
LEMMA 3: If $h \in N$ and $n \in \mathbb{S}_{m}$, then $V_{h n}(m) \equiv V_{h}(m)(\bmod n)$.

Proof: Let us rewrite the result established in $[13$, Cor. 7$]$ as

$$
V_{h n}(m)=\sum_{i=0}^{[h / 2]} C_{h, i} V_{n}^{h-2 i}(m) \quad(n \text { odd }) .
$$

By hypothesis, (4.25) and (1.6), we can write

$$
V_{h n}(m)=\sum_{i=0}^{[h / 2]} C_{h, i} V_{n}^{h-2 i}(m) \equiv \sum_{i=0}^{[h / 2]} C_{h, i} m^{h-2 i}=V_{h}(m)(\bmod n) . \quad \text { Q.E.D. }
$$

THEOREM 6: If an odd composite $n$ passes the $m^{\text {th }}$ test, then it passes also the $V_{2 k+1}(m)^{\text {th }}$ tests $(k=1,2, \ldots)$.

Proof: From Lemma 2, Lemma 3 can read: If $V_{n}(m) \equiv m(\bmod n)$, then

$$
V_{n}\left(V_{2 k+1}(m)\right)=V_{n(2 k+1)}(m) \equiv V_{2 k+1}(m)(\bmod n) \text {. Q.E.D. }
$$

As particular cases, we see that

- if $n$ passes the $1^{\text {st }}$ test $(m=1)$, then it passes also the tests for $m=4,11,29,76$, $199,521,1364, \ldots$

- if $n$ passes the $2^{\text {nd }}$ test ( $m=2$ ), then it passes also for $m=14,82,478,2786, \ldots$

- if $n$ passes the $3^{\text {rd }}$ test $(m=3)$, then it passes also for $m=36,393,4287,46764, \ldots$

- if $n$ passes the $4^{\text {th }}$ test ( $m=4$ ), then it passes also for $m=76,1364, \ldots$ (cf. the tests passed for $m=1$ ).

\section{Conclusion}

Public-key cryptosystems make use of primes having approximately 100 digits, so we wish to conclude this paper with two questions.

Pessimist's question: "Do odd composites $n \leq 10^{100}$ exist which are $m$-F.Psps. for all values of $m \leq n-1$ ?"

If such numbers exist, they will never reveal their compositeness under our test.

Optimist's question: "Let $M^{*}$ be the maximum number of consecutive tests ( $m=1$, $2, \ldots, M^{*}$ ) passed by any odd composite $n \leq 10^{100}$. Is $M^{*}$ comparatively small (say $M^{*}$ $\leq 50)$ ?

If the answer is in affirmative, then the method proposed in Sec. 3 can readily find primes for cryptographic purposes. The calculation time is slightly less than that 
required by the method proposed by Solovay \& Strassen [14] for finding numbers that are prime with probability greater than or equal to $1-1 / 2^{M^{*}}$.

The authors offer a prize of 50,000 Italian Lire to the first person who communicates to them an odd composite (below $10^{100}$ ) which is an $m$-F.Psp. for $m=$ $1,2, \ldots, 8$. Of course, at least one of its factors is also requested. A decuple prize is offered to the first person who sends to them a proof that no such number exists.

A table of 1-F.Psps to $10^{8}$ was compiled by the authors. It will be sent, free of charges, upon request.

\section{References}

[1] H.Riesel, Prime Numbers and Computer Methods for Factorization . Boston: Birkhäuser Inc., 1985.

[2] M.Bicknell, "A Primer on the Pell Sequence and Related Sequences", The Fibonacci Quarterly , vol.13, pp. 345-349, no.4, 1975.

[3] O.Brugia, P.Filipponi, "Waring Formulae and Certain Combinatorial Identities", Fondaz. Ugo Bordoni Techn. Rep. 3B5986, Oct. 1986.

[4] A.Di Porto, P.Filipponi, "More on the Fibonacci Pseudoprimes", Fondaz.Ugo Bordoni Techn. Rep. 3t0687, May 1987. The Fibonacci Quarterly (to appear).

[5] A.Di Porto, P.Filipponi, "Un Metodo di Prova di Primalità Basato sulle Proprietà dei Numeri di Lucas Generalizzati", Proc. of the Primo Simposio Nazionale su: Stato e Prospettive della Ricerca Crittografica in Italia , Roma, Oct. 1987, pp. 141-146.

[6] Bro. A.Brousseau, An Introduction to Fibonacci Discovery . Santa Clara (Cal.): The Fibonacci Association, 1965.

[7] L.Carlitz, "A Note on Fibonacci Numbers", The Fibonacci Quarterly, vol. 2, pp. 15-28, no.1, 1964.

[8] D.Jarden, Recurring Sequences, $3^{\text {rd }}$ ed., Jerusalem : Riveon Lematematika, 1973.

[9] V.E.Hoggatt, Jr., M.Bicknell, "Some Congruences of the Fibonacci Numbers Modulo a Prime $P$ ", Math. Magazine, vol. 47, pp. 210-214, no.3, 1974.

[10] V.E.Hoggatt, Jr., Fibonacci and Lucas Numbers, Boston: Houghton Mifflin Co., 1969.

[11] V.E.Hoggatt, Ir., G.E.Bergum, "Divisibility and Congruence Relations", The Fibonacci Quarterly, vol. 12, pp. 189-195, no. 2, 1974.

[12] P.Filipponi:"On the Divisibility of Certain Generalized Fibonacci Numbers by Their Subscripts", Proc. XIII Congresso Unione Matematica Italiana, Torino, Sept. 1987, Sezione VII-18.

[13] Jin-Zai Lee, Jia-Sheng Lee, "Some Properties of the Sequence $\left\{W_{n}(a, b ; p, q)\right\}$ ", The Fibonacci Quarterly, vol. 25, pp. 268-278, 283, no. 3, 1987.

[14] R.Solovay, V.Strassen, "A Fast Monte-Carlo Test for Primality", SIAM Journal on Comput., vol. 6, pp. 84-85, no.1, 1977. 